Lichun WANG (Diepenbeek)

\title{
BAYES AND EMPIRICAL BAYES TESTS FOR THE LIFE PARAMETER
}

Abstract. We study the one-sided testing problem for the exponential distribution via the empirical Bayes (EB) approach. Under a weighted linear loss function, a Bayes test is established. Using the past samples, we construct an EB test and exhibit its optimal rate of convergence. When the past samples are not directly observable, we work out an EB test by using the deconvolution kernel method and obtain its asymptotic optimality.

1. Introduction. Let us consider the problem of testing the hypothesis

$$
H_{0}: \theta \leq \theta_{0} \leftrightarrow H_{1}: \theta>\theta_{0}
$$

under the exponential distribution

$$
f_{\theta}(x)=\frac{1}{\theta} \exp \left(-\frac{x}{\theta}\right), \quad x>0,
$$

where $f_{\theta}(x)$ denotes the conditional probability density function (pdf) of a random variable (r.v.) $X$ given $\theta$.

In practice, the distribution (1.2) appears very often and is important, and it can be used to describe many models of survival analysis, reliability theory, engineering and environmental sciences. Usually, the data observed from this distribution is the lifetime of an individual in survival analysis and reliability problems. Since the expectation of $X$ is equal to $\theta$, we call $\theta$ the life parameter.

2000 Mathematics Subject Classification: Primary 62C12; Secondary 62F12.

Key words and phrases: empirical Bayes, asymptotic optimality, rate of convergence, exponential distribution.

Financial support from the IAP research network (\# P5/24) of the Belgian Government (Belgian Science Policy) and NNSF of China (No. 10271001) is gratefully acknowledged. 
We adopt a weighted linear error loss function defined as follows:

$$
L\left(\theta, d_{m}\right)=(1-m) \frac{\theta-\theta_{0}}{\theta} I_{\left[\theta>\theta_{0}\right]}+m \frac{\theta_{0}-\theta}{\theta} I_{\left[\theta_{0} \geq \theta\right]},
$$

where $d_{m}$ denotes an action in favor of $H_{m}(m=0,1)$, and $I_{[A]}$ is the indicator of the set $A$. Obviously, the loss function (1.3) is more reasonable for the life parameter than the ordinary linear loss since it can remove the influence of the measurement unit. Suppose the parameter $\theta$ is distributed according to a prior $G(\theta)$ with support on $\Theta=(0, \infty)$.

Let

$$
\delta(x)=P\left(\text { accepting } H_{0} \mid X=x\right) .
$$

Then the Bayes risk of the test $\delta(x)$ is given by

$$
\begin{aligned}
R(\delta(x), G(\theta)) & \\
& =\int_{0}^{\infty} \int_{\Theta}\left[L\left(\theta, d_{0}\right) \delta(x)+L\left(\theta, d_{1}\right)(1-\delta(x))\right] f_{\theta}(x) d G(\theta) d x \\
& =\int_{0}^{\infty} \alpha(x) \delta(x) d x+\int_{\Theta} \theta^{-1}\left(\theta_{0}-\theta\right) I_{\left[\theta_{0} \geq \theta\right]} d G(\theta)
\end{aligned}
$$

with

$$
\alpha(x)=\int_{\Theta} \theta^{-1}\left(\theta-\theta_{0}\right) f_{\theta}(x) d G(\theta)=f(x)+\theta_{0} f^{(1)}(x),
$$

where $f(x)=\int_{\Theta} f_{\theta}(x) d G(\theta)$ is the marginal pdf of $X$, and $f^{(1)}(x)$ denotes the derivative of $f(x)$.

Hence, the best Bayes test minimizing $R(\delta(x), G(\theta))$ would have the form

$$
\delta_{G}(x)= \begin{cases}1, & \alpha(x) \leq 0 \\ 0, & \alpha(x)>0\end{cases}
$$

The minimum Bayes risk is

$$
R\left(\delta_{G}(x), G(\theta)\right)=\int_{0}^{\infty} \alpha(x) \delta_{G}(x) d x+\int_{\Theta} \theta^{-1}\left(\theta_{0}-\theta\right) I_{\left[\theta_{0} \geq \theta\right]} d G(\theta) .
$$

Define $\beta(x)=\alpha(x) / f(x)$. Then by the Cauchy-Schwarz inequality, it is easy to see that $\beta^{(1)}(x) \geq 0$. Assume that the prior $G(\theta)$ satisfies

$$
\lim _{x \rightarrow \infty} \beta(x)>0>\lim _{x \rightarrow 0} \beta(x) .
$$

Obviously, (1.9) implies that $G(\theta)$ is nondegenerate and $\beta(x)$ is a strictly increasing function. Therefore, by the continuity of $\beta(x)$, there exists a unique point $a_{G}$ such that $\beta\left(a_{G}\right)=0$. Then

$$
\delta_{G}(x)=\left\{\begin{array}{ll}
1, & \alpha(x) \leq 0 \\
0, & \alpha(x)>0
\end{array}=\left\{\begin{array}{ll}
1, & \beta(x) \leq 0 \\
0, & \beta(x)>0
\end{array}= \begin{cases}1, & x \leq a_{G} \\
0, & x>a_{G} .\end{cases}\right.\right.
$$


REMARK 1. As an application, suppose that the life parameter $\theta$ has a prior pdf

$$
g(\theta)=\frac{d G(\theta)}{d \theta}=\frac{1}{\Gamma(b-2)}\left(\frac{1}{\theta}\right)^{b-1} \exp \left(-\frac{1}{\theta}\right), \quad b>2, \theta>0 .
$$

For example, let $b=3$. Then $f(x)=(x+1)^{-2}, x>0$. It is readily seen that $\beta(x)=1-2 \theta_{0}(x+1)^{-1}$ and $a_{G}=2 \theta_{0}-1$, so we get

$$
\delta_{G}(x)= \begin{cases}1, & x \leq 2 \theta_{0}-1 \\ 0, & x>2 \theta_{0}-1\end{cases}
$$

But in many situations, since the prior $G(\theta)$ may be unknown to us, the Bayes test $\delta_{G}(x)$ of (1.10) is unavailable. As an alternative we can use the EB approach to estimate $\alpha(x)$ in (1.6) so as to obtain an EB test $\delta_{n}(x)$.

EB approach was first introduced to statistical problems by Robbins $[6,7]$ and has been applied in a wide range of paradigms and to numerous real-life problems. Some earlier papers, such as [2], discussed the EB testing problem for the discrete case, whereas [8] and [9] concentrated on the EB testing problems in the continuous one-parameter exponential family. Recently the author of [5], who continues the research of [3], [9] and [4], has considered the EB testing problem in a positive exponential family, and obtained a better rate of convergence under the assumption that the critical point $a_{G}$ is within some known compact interval.

In this paper, we discuss the EB testing problem for the life parameter in the exponential distribution, firstly, under the condition that the past samples are not contaminated, and secondly, that they are contaminated.

The rest of the paper is organized as follows. In Section 2 we propose an EB test and exhibit the optimal convergence rate. In Section 3 we discuss the case when the past samples are contaminated by a normal error variable.

2. Empirical Bayes test and rate of convergence. In the empirical Bayes framework, we usually make the following assumptions: let $\left(X_{i}, \theta_{i}\right)$, $i=1,2, \ldots$, be independent identically distributed (i.i.d.) copies of $(X, \theta)$, where $X_{i}, i=1,2, \ldots$, are observable, but $\theta_{i}, i=1,2, \ldots$, are not. At time $n+1$, we observe $X \widehat{=} X_{n+1}$ and plan to test the hypothesis: $H_{0}: \theta \leq \theta_{0} \leftrightarrow$ $H_{1}: \theta>\theta_{0}$, where $\theta \widehat{=} \theta_{n+1}$. Usually, the $\left(X_{1}, \ldots, X_{n}\right)$ denote the past samples and $X$ is the present sample.

In order to construct an EB test, we use two kernel functions $K_{l}(x)(l=$ $0,1)$ which are Borel measurable bounded real functions vanishing off $(0,1)$ such that

$$
\int_{0}^{1} x^{p} K_{l}(x) d x= \begin{cases}1, & p=l, \\ 0, & p \neq l, p=1, \ldots, s-1, \quad \int_{0}^{1} x^{s}\left|K_{l}(x)\right| d x<\infty .\end{cases}
$$


where $s \geq 2$ is an arbitrary but fixed integer. It is easy to show that there exist some polynomials which satisfy the above conditions.

Define a kernel estimator of $f(x)$ and $f^{(1)}(x)$, respectively, as

$$
\begin{aligned}
f_{n}(x) & =\frac{1}{n h_{n}} \sum_{i=1}^{n} K_{0}\left(\frac{X_{i}-x}{h_{n}}\right), \\
f_{n}^{(1)}(x) & =\frac{1}{n h_{n}^{2}} \sum_{i=1}^{n} K_{1}\left(\frac{X_{i}-x}{h_{n}}\right),
\end{aligned}
$$

where $0<h_{n} \rightarrow 0(n \rightarrow \infty)$ denotes the bandwidth. Then we have

$$
\alpha_{n}(x)=f_{n}(x)+\theta_{0} f_{n}^{(1)}(x) .
$$

We consider those prior distributions $G(\theta)$ for which $G(\theta) \in \mathcal{F}=\{G(\theta)$ : $0<A_{1} \leq a_{G} \leq A_{2}<\infty, A_{1}, A_{2}$ are known constants $\}$. Since $G(\theta) \in \mathcal{F}$, taking into account the Bayes test (1.10), we propose an EB test defined as follows:

$$
\delta_{n}(x)=\left\{\begin{array}{l}
1, x<A_{1} \text { or }\left(A_{1} \leq x \leq A_{2} \text { and } \alpha_{n}(x) \leq 0\right), \\
0, x>A_{2} \text { or }\left(A_{1} \leq x \leq A_{2} \text { and } \alpha_{n}(x)>0\right) .
\end{array}\right.
$$

Hence, the Bayes risk of $\delta_{n}(x)$ is

$$
R\left(\delta_{n}(x), G(\theta)\right)=\int_{0}^{\infty} \alpha(x) E_{n}\left[\delta_{n}(x)\right] d x+\int_{\Theta} \theta^{-1}\left(\theta_{0}-\theta\right) I_{\left[\theta_{0} \geq \theta\right]} d G(\theta),
$$

where $E_{n}$ denotes the expectation with respect to the joint distribution of $\left(X_{1}, \ldots, X_{n}\right)$.

By definition, the EB test $\delta_{n}(x)$ is said to be asymptotically optimal relative to the prior $G(\theta)$ if $R\left(\delta_{n}(x), G(\theta)\right)-R\left(\delta_{G}(x), G(\theta)\right)=o(1)$. If for some $q>0, R\left(\delta_{n}(x), G(\theta)\right)-R\left(\delta_{G}(x), G(\theta)\right)=O\left(n^{-q}\right)$, then the EB test $\delta_{n}(x)$ is said to have convergence rate $O\left(n^{-q}\right)$.

REMARK 2. Usually, there are two different forms of EB test in the literature. One form, used in $[2,3]$ and some other papers, suggests the following test for the above problem:

$$
\delta_{n}(x)= \begin{cases}1, & \alpha_{n}(x) \leq 0 \\ 0, & \alpha_{n}(x)>0 .\end{cases}
$$

It is not assumed that the critical point $a_{G}$ is in the compact interval $\left[A_{1}, A_{2}\right]$, accordingly, the monotonicity of $\beta(x)$ is not considered. The other form, i.e. (2.3), appeared in [9], [4], and [5], and is named the monotone EB test. In the author's opinion, the EB test $\delta_{n}(x)(2.3)$ is relatively reasonable since it divides the interval $(0, \infty)$ into three parts, but one will have to make some additional assumption about the critical point. 
Lemma 1. Let $f_{n}^{(l)}(x)(l=0,1)$ be as defined in $(2.1)$. If $E\left(\theta^{-(s+1)}\right)<\infty$ for some integer $s \geq 2$, then

$$
\left|E_{n} f_{n}^{(l)}(x)-f^{(l)}(x)\right|=O\left(h_{n}^{s-l}\right), \quad l=0,1 .
$$

Proof. Expanding $f\left(x+u h_{n}\right)$ at $x$ and using the properties of $K_{l}(x)$, we obtain

$$
\begin{aligned}
& E_{n} f_{n}^{(l)}(x)=\frac{1}{h_{n}^{l+1}} \int_{0}^{\infty} K_{l}\left(\frac{y-x}{h_{n}}\right) f(y) d y=\frac{1}{h_{n}^{l}} \int_{0}^{1} K_{l}(u) f\left(x+u h_{n}\right) d u \\
& =\frac{1}{h_{n}^{l}} \int_{0}^{1}\left[f(x)+\cdots+\frac{f^{(s-1)}(x)}{(s-1) !}\left(u h_{n}\right)^{s-1}+\frac{f^{(s)}\left(x+\xi u h_{n}\right)}{s !}\left(u h_{n}\right)^{s}\right] \\
& \quad \times K_{l}(u) d u \\
& =f^{(l)}(x)+\frac{h_{n}^{s-l}}{s !} \int_{0}^{1} K_{l}(u) f^{(s)}\left(x+\xi u h_{n}\right) u^{s} d u, \quad 0<\xi<1 .
\end{aligned}
$$

As $E\left(\theta^{-(s+1)}\right)<\infty$, we have $\sup _{x}\left|f^{(s)}(x)\right|<\infty$. So Lemma 1 is proved.

We now represent $\alpha(x)$ by

$$
\begin{aligned}
\alpha(x) & =\frac{1}{n h_{n}} \sum_{i=1}^{n} K_{0}\left(\frac{X_{i}-x}{h_{n}}\right)+\theta_{0} \frac{1}{n h_{n}^{2}} \sum_{i=1}^{n} K_{1}\left(\frac{X_{i}-x}{h_{n}}\right) \\
& \widehat{=} \frac{1}{n} \sum_{i=1}^{n} R\left(x, X_{i}, h_{n}\right)
\end{aligned}
$$

with

$$
R\left(x, X_{i}, h_{n}\right)=\frac{1}{h_{n}} K_{0}\left(\frac{X_{i}-x}{h_{n}}\right)+\frac{\theta_{0}}{h_{n}^{2}} K_{1}\left(\frac{X_{i}-x}{h_{n}}\right) .
$$

Note that $R\left(x, X_{i}, h_{n}\right)(i=1, \ldots, n)$ are i.i.d. r.v. such that

$$
\left|R\left(x, X_{i}, h_{n}\right)-E_{n} R\left(x, X_{i}, h_{n}\right)\right| \leq 2\left(\frac{M_{0}}{h_{n}}+\frac{\theta_{0}}{h_{n}^{2}} M_{1}\right)
$$

and

(2.9) $\operatorname{Var}\left(R\left(x, X_{i}, h_{n}\right)\right)$

$$
\begin{aligned}
& \leq \frac{2}{h_{n}^{2}} \operatorname{Var}\left(K_{0}\left(\frac{X_{i}-x}{h_{n}}\right)\right)+\frac{2 \theta_{0}^{2}}{h_{n}^{4}} \operatorname{Var}\left(K_{1}\left(\frac{X_{i}-x}{h_{n}}\right)\right) \\
& \leq \frac{2}{h_{n}^{2}} E\left(K_{0}\left(\frac{X_{i}-x}{h_{n}}\right)\right)^{2}+\frac{2 \theta_{0}^{2}}{h_{n}^{4}} E\left(K_{1}\left(\frac{X_{i}-x}{h_{n}}\right)\right)^{2}
\end{aligned}
$$




$$
\begin{aligned}
& =\frac{2}{h_{n}} \int_{0}^{1} K_{0}^{2}(u) f\left(x+u h_{n}\right) d u+\frac{2 \theta_{0}^{2}}{h_{n}^{3}} \int_{0}^{1} K_{1}^{2}(u) f\left(x+u h_{n}\right) d u \\
& \leq 2 c\left(h_{n}^{-1}+h_{n}^{-3}\right),
\end{aligned}
$$

where $M_{l}>0(l=0,1)$ denotes the bound of the kernel function $K_{l}(x)(l=$ $0,1)$, and $c$ is a positive constant that does not depend on $n$.

Define $A_{G}=\min _{A_{1} \leq x \leq A_{2}} f(x)$, and let $a_{1 n}<a_{G}<a_{2 n}$ be the point such that $-\beta\left(a_{1 n}\right)=2 c h_{n}^{s-1} / A_{G}=\beta\left(a_{2 n}\right)$. Since $\beta(x)$ is continuous, we know that $\lim _{n \rightarrow \infty} a_{1 n}=\lim _{n \rightarrow \infty} a_{2 n}=a_{G}$.

It follows from (1.8) and (2.4) that

$$
\begin{aligned}
0 \leq & R\left(\delta_{n}(x), G(\theta)\right)-R\left(\delta_{G}(x), G(\theta)\right) \\
= & \int_{0}^{\infty}\left[E_{n} \delta_{n}(x)-\delta_{G}(x)\right] \alpha(x) d x \\
= & \int_{A_{1}}^{a_{G}}\left[P\left(\alpha_{n}(x) \leq 0\right)-1\right] \alpha(x) d x+\int_{a_{G}}^{A_{2}} P\left(\alpha_{n}(x) \leq 0\right) \alpha(x) d x \\
= & -\int_{A_{1}}^{a_{1 n}} P\left(\alpha_{n}(x)>0\right) \alpha(x) d x-\int_{a_{1 n}}^{a_{G}} P\left(\alpha_{n}(x)>0\right) \alpha(x) d x \\
& +\int_{a_{G}}^{a_{2 n}} P\left(\alpha_{n}(x) \leq 0\right) \alpha(x) d x+\int_{a_{2 n}}^{A_{2}} P\left(\alpha_{n}(x) \leq 0\right) \alpha(x) d x \\
\widehat{=} & \sum_{i}^{4} I_{i} .
\end{aligned}
$$

It is easy to see that

$$
I_{2} \leq-\int_{a_{1 n}}^{a_{G}} \alpha(x) d x \leq-\beta\left(a_{1 n}\right) \int_{a_{1 n}}^{a_{G}} f(x) d x=O\left(h_{n}^{s-1}\right) .
$$

Similarly, we get

$$
I_{3}=O\left(h_{n}^{s-1}\right)
$$

Note that

$$
\alpha(x) \leq \beta\left(a_{1 n}\right) f(x) \leq \beta\left(a_{1 n}\right) A_{G}=-2 c h_{n}^{s-1}, \quad A_{1} \leq x \leq a_{1 n} .
$$

Furthermore, by Lemma $1, E_{n} \alpha_{n}(x) \leq \alpha(x)+c h_{n}^{s-1} \leq \alpha(x) / 2$. Hence, for $A_{1} \leq x \leq a_{1 n}$, we have

$$
P\left(\alpha_{n}(x)>0\right) \leq P\left(\alpha_{n}(x)-E_{n} \alpha_{n}(x) \geq-\frac{1}{2} \alpha(x)\right) .
$$


Combining (2.8), (2.9) and (2.14), by Bernstein's inequality, we obtain

$$
\begin{aligned}
& \leq 2 \exp \left\{\frac{-n^{2}(-\alpha(x) / 2)^{2}}{2 \operatorname{Var}\left(\sum_{i=1}^{n} R\left(x, X_{i}, h_{n}\right)\right)+4\left(M_{0} / h_{n}+\theta_{0} M_{1} / h_{n}^{2}\right)(-n \alpha(x) / 2) / 3}\right\} \\
& =2 \exp \left\{\frac{-n(\alpha(x))^{2} / 8}{\operatorname{Var}\left(R\left(x, X_{i}, h_{n}\right)\right)+\left(M_{0} / h_{n}+\theta_{0} M_{1} / h_{n}^{2}\right)|\alpha(x)| / 3}\right\} \\
& \leq 2 \exp \left\{-\frac{n h_{n}^{3}}{8} \times \frac{A_{G}^{2}(\beta(x))^{2}}{2 c h_{n}^{2}+2 c+\left(M_{0} h_{n}^{2}+\theta_{0} M_{1} h_{n}\right) E\left(\theta^{-1}\right)\left|\beta\left(A_{1}\right)\right| / 3}\right\} \\
& \widehat{=} 2 \exp \left\{-n h_{n}^{3} J\left(h_{n}\right)(\beta(x))^{2}\right\}, \quad A_{1} \leq x \leq a_{1 n},
\end{aligned}
$$

where

$$
J\left(h_{n}\right)=A_{G}^{2} /\left[8\left(2 c h_{n}^{2}+2 c+\left(M_{0} h_{n}^{2}+\theta_{0} M_{1} h_{n}\right) E\left(\theta^{-1}\right)\left|\beta\left(A_{1}\right)\right| / 3\right)\right] .
$$

From (2.10) and (2.15), we have

$$
\begin{aligned}
& I_{1} \leq-2 \int_{A_{1}}^{a_{1 n}} \exp \left\{-n h_{n}^{3} J\left(h_{n}\right)(\beta(x))^{2}\right\} \beta(x) f(x) d x \\
& \leq-2 \sup _{A_{1} \leq x \leq A_{2}}\left[\frac{f(x)}{\beta^{(1)}(x)}\right] \int_{A_{1}}^{a_{1 n}} \exp \left\{-n h_{n}^{3} J\left(h_{n}\right)(\beta(x))^{2}\right\} \beta(x) \beta^{(1)}(x) d x \\
& =O\left(\frac{1}{n h_{n}^{3}}\right) .
\end{aligned}
$$

Similarly, we get

$$
I_{4}=O\left(\frac{1}{n h_{n}^{3}}\right) .
$$

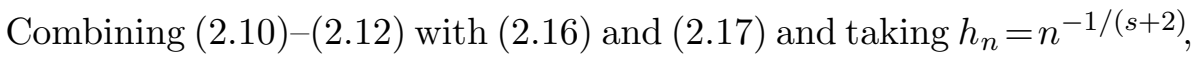
we conclude that

$$
0 \leq R\left(\delta_{n}(x), G(\theta)\right)-R\left(\delta_{G}(x), G(\theta)\right)=O\left(n^{-(s-1) /(s+2)}\right) .
$$

Hence, we can state the following theorem.

Theorem 1. Let the Bayes test $\delta_{G}(x)$ and $E B$ test $\delta_{n}(x)$ be as defined in (1.10) and (2.3), respectively. If $G(\theta) \in \mathcal{F}$ (defined before) and $E\left(\theta^{-(s+1)}\right)$ $<\infty$ for some integer $s \geq 2$, then choosing $h_{n}=n^{-1 /(s+2)}$, we have

$$
R\left(\delta_{n}(x), G(\theta)\right)-R\left(\delta_{G}(x), G(\theta)\right)=O\left(n^{-(s-1) /(s+2)}\right) .
$$

REMARK 3. If we use the linear loss function

$$
L\left(\theta, d_{m}\right)=(1-m)\left(\theta-\theta_{0}\right) I_{\left[\theta>\theta_{0}\right]}+m\left(\theta_{0}-\theta\right) I_{\left[\theta_{0} \geq \theta\right]}, \quad m=0,1,
$$


then it is not difficult to see that

$$
\begin{aligned}
\alpha(x) & =\int_{\Theta}\left(\theta-\theta_{0}\right) f_{\theta}(x) d G(\theta)=\int_{\Theta} \exp (-x / \theta) d G(\theta)-\theta_{0} f(x) \\
& =\int_{x}^{\infty} f(y) d y-\theta_{0} f(x) .
\end{aligned}
$$

Thus, we only need to estimate $f(x)$. Following a proof analogous to the preceding discussion, we can improve the rate of convergence $O\left(n^{-(s-1) /(s+2)}\right)$ to the best rate $O\left(n^{-s /(s+1)}\right)$ for testing hypothesis (1.1).

To the best of our knowledge, the convergence rate $o\left(n^{-1}\right)$ cannot be attained with any EB test for any nondiscrete density. Therefore, it is very hard to improve the rate of convergence $O\left(n^{-(s-1) /(s+2)}\right)$ under the weighted linear loss function (1.3) since it tends to be $O\left(n^{-1}\right)$ as $s$ gets larger.

3. The case when the data are contaminated. Suppose that the past samples $\left(X_{1}, \ldots, X_{n}\right)$ are contaminated due to measurement or the nature of environment, and one can only observe

$$
Y_{j}=X_{j}+\varepsilon, \quad j=1, \ldots, n,
$$

where the error variable $\varepsilon$ has a known distribution $F_{\varepsilon}$. Furthermore, assume that $\varepsilon$ and $X_{j}$ are independent. Problems with contaminating errors exist in many different fields (e.g., biostatistics, electrophoresis) and have been widely studied. The authors of [10] and [11] discussed the EB estimation for the continuous one-parameter exponential family with errors in variables under the squared loss function, and obtained asymptotic optimality and uniform rate of convergence for the proposed EB estimator over a class of prior distributions.

In this section, we discuss the asymptotic behavior of EB tests under the assumption that $\varepsilon \sim N\left(0, \sigma^{2}\right)$ with $\sigma^{2}$ known.

Similarly to [1], using the deconvolution kernel method, we make the following assumptions on the kernel:

(1) $k(x)$ is bounded, continuous, and $\int_{-\infty}^{\infty}|x|^{s}|k(x)| d x<\infty$.

(2) The Fourier transform $\phi_{k}(t)$ of $k(x)$ is a symmetric function satisfying $\phi_{k}(t)=1+O\left(|t|^{s}\right)$ as $t \rightarrow 0$.

(3) $\phi_{k}(t)=0$ for $|t| \geq 1$.

Here $s \geq 2$ is an arbitrary but fixed integer and

$$
\phi_{k}(t)=\int_{-\infty}^{\infty} \exp (i t x) k(x) d x
$$

By assumptions (1)-(3), we can easily get

$$
\int k(x) d x=1, \quad \int x^{p} k(x) d x=0, \quad p=1, \ldots, s-1, \quad \int|x|^{s}|k(x)| d x<\infty .
$$


Noting that $f(x)=(2 \pi)^{-1} \int_{-\infty}^{\infty} \exp (-i t x) \phi_{Y}(t) \exp \left(\sigma^{2} t^{2} / 2\right) d t$, we define an estimator of $f^{(l)}(x)(l=0,1)$ by

$$
\widehat{f}_{n}^{(l)}(x)=\frac{1}{2 \pi} \int_{-\infty}^{\infty} \exp (-i t x)(-i t)^{l} \phi_{k}\left(t b_{n}\right) \widehat{\phi}_{n}(t) \exp \left(\sigma^{2} t^{2} / 2\right) d t
$$

where $0<b_{n} \rightarrow 0$ as $n \rightarrow \infty$, and $\widehat{\phi}_{n}(t)=n^{-1} \sum_{j=1}^{n} \exp \left(i t Y_{j}\right)$ is an estimator of the characteristic function (c.f.) $\phi_{Y}(t)$ of the r.v. $Y$, which is called the empirical c.f. of $Y$.

Rewrite (3.2) as a kernel type estimator

$$
\widehat{f}_{n}^{(l)}(x)=\frac{1}{n b_{n}^{l+1}} \sum_{j=1}^{n} k_{n l}\left(\frac{x-Y_{j}}{b_{n}}\right), \quad l=0,1,
$$

where

$$
k_{n l}(x)=\frac{1}{2 \pi} \int_{-\infty}^{\infty} \exp (-i t x)(-i t)^{l} \phi_{k}(t) \exp \left(\sigma^{2} t^{2} /\left(2 b_{n}^{2}\right)\right) d t .
$$

Hence, under the assumption that $G(\theta) \in \mathcal{F}$, we propose the following EB test:

$$
\widehat{\delta}_{n}(x)= \begin{cases}1, & x<A_{1} \text { or }\left(A_{1} \leq x \leq A_{2} \text { and } \widehat{\alpha}_{n}(x) \leq 0\right) \\ 0, & x>A_{2} \text { or }\left(A_{1} \leq x \leq A_{2} \text { and } \widehat{\alpha}_{n}(x)>0\right)\end{cases}
$$

where

$$
\widehat{\alpha}_{n}(x)=\widehat{f}_{n}(x)+\theta_{0} \widehat{f}_{n}^{(1)}(x) \widehat{=} \frac{1}{n} \sum_{j=1}^{n} V\left(x, Y_{j}, b_{n}\right)
$$

with i.i.d. r.v.

$$
V\left(x, Y_{j}, b_{n}\right)=\frac{1}{b_{n}} k_{n 0}\left(\frac{x-Y_{j}}{b_{n}}\right)+\frac{\theta_{0}}{b_{n}^{2}} k_{n 1}\left(\frac{x-Y_{j}}{b_{n}}\right) .
$$

Lemma 2. Let $\widehat{f}_{n}^{(l)}(x)(l=0,1)$ be as in (3.2). If $G(\theta) \in \mathcal{F}$ and $E\left(\theta^{-(s+1)}\right)$ $<\infty$ for some integer $s \geq 2$, where $\mathcal{F}$ is as before, then

(a) $\left|E_{n} \widehat{f}_{n}^{(l)}(x)-f(x)\right|=O\left(b_{n}^{s-l}\right), l=0,1$;

(b) $\left|E_{n} \widehat{\alpha}_{n}(x)-\alpha(x)\right|=O\left(b_{n}^{s-1}\right)$;

(c) $\left|V\left(x, Y_{j}, b_{n}\right)-E_{n} V\left(x, Y_{j}, b_{n}\right)\right| \leq 2\left(b_{n}^{-1}+\theta_{0} b_{n}^{-2}\right) O\left(\exp \left(\sigma^{2} b_{n}^{-2} / 2\right)\right)$;

(d) $\operatorname{Var}\left(V\left(x, Y_{j}, b_{n}\right)\right)=2\left(b_{n}^{-2}+\theta_{0}^{2} b_{n}^{-4}\right) O\left(\exp \left(\sigma^{2} b_{n}^{-2}\right)\right)$.

Here $E_{n}$ denotes the expectation with respect to the joint distribution of $\left(Y_{1}, \ldots, Y_{n}\right)$. 
Proof. (a) By assumptions (1)-(3) on $k(x)$, we have

$$
\begin{aligned}
& E_{n} \widehat{f}_{n}^{(l)}(x)-f^{(l)}(x)=\int f^{(l)}\left(x-b_{n} y\right) k(y) d y-f^{(l)}(x) \\
= & \int\left[f^{(l)}(x)+\cdots+\frac{f^{(s-1)}(x)\left(-b_{n} y\right)^{s-l-1}}{(s-l-1) !}+\frac{f^{(s)}\left(x-\xi_{1} b_{n} y\right)\left(-b_{n} y\right)^{s-l}}{(s-l) !}\right] \\
& \times k(y) d y-f^{(l)}(x) \\
= & \int \frac{f^{(s)}\left(x-\xi_{1} b_{n} y\right)\left(-b_{n} y\right)^{s-l}}{(s-l) !} k(y) d y, \quad 0<\xi_{1}<1 .
\end{aligned}
$$

Hence, (a) holds under the condition that $E\left(\theta^{-(s+1)}\right)<\infty$.

(b) is obvious.

(c) For $l=0,1$, by Theorem 1 of [1], we know that

$$
\begin{aligned}
\left|k_{n l}(x)\right|^{2} & \leq \frac{1}{(2 \pi)^{2}}\left(\int\left|\phi_{k}(t) t^{l}\right| \exp \left(\sigma^{2} t^{2} /\left(2 b_{n}^{2}\right)\right) d t\right)^{2} \\
& =O\left(\exp \left(\sigma^{2} b_{n}^{-2}\right)\right)
\end{aligned}
$$

by letting $\beta=2$ and $\beta_{0}=0$ in [1]. Thus,

$$
\left|V\left(x, Y_{j}, b_{n}\right)\right|=b_{n}^{-1} O\left(\exp \left(\sigma^{2} b_{n}^{-2} / 2\right)\right)+\theta_{0} b_{n}^{-2} O\left(\exp \left(\sigma^{2} b_{n}^{-2} / 2\right)\right),
$$

and (c) is proved.

(d) Noting that

$$
\operatorname{Var}\left(k_{n l}\left(\frac{x-Y_{j}}{b_{n}}\right)\right)=n b_{n}^{2 l+2} \operatorname{Var}\left(\widehat{f}_{n}(x)\right)=O\left(\exp \left(\sigma^{2} b_{n}^{-2}\right)\right),
$$

we find that $(\mathrm{d})$ is true.

Using Lemma 2, by mimicking the steps in Section 2, we have

$$
\begin{aligned}
0 & \leq R\left(\widehat{\delta}_{n}(x), G(\theta)\right)-R\left(\delta_{G}(x), G(\theta)\right) \\
& =\frac{1}{n b_{n}^{4}} O\left(\exp \left(\sigma^{2} b_{n}^{-2}\right)\right)+O\left(b_{n}^{s-1}\right) .
\end{aligned}
$$

Taking $b_{n}=\sqrt{2 \sigma^{2}}(\log n)^{-1 / 2}$, we obtain $\left(n b_{n}^{4}\right)^{-1} O\left(\exp \left(\sigma^{2} b_{n}^{-2}\right)\right)=o\left(n^{-\tau}\right)$, where $\tau>0$ can be arbitrarily close to $1 / 2$. Therefore, the EB test $\widehat{\delta}_{n}(x)$ of (3.5) is asymptotically optimal under the conditions that $G(\theta) \in \mathcal{F}$ and $E\left(\theta^{-(s+1)}\right)<\infty$.

REMARK 4. As described in [1], it is extremely difficult to solve deconvolution problems when the error distributions are normal or Cauchy (called supersmooth distributions). Actually, if the error has gamma distribution, which belongs to ordinary smooth ones, then we can obtain a higher rate of convergence employing the idea of $[1]$ used in $[10,11]$. However, normal errors deserve more attention due to their importance. 
Acknowledgements. The author is indebted to an anonymous referee for his valuable suggestions.

\section{References}

[1] J. Fan, On the optimal rates of convergence for nonparametric deconvolution problems, Ann. Statist. 3 (1991), 1257-1272.

[2] M. V. Johns Jr. and J. R. Van Ryzin, Convergence rates for empirical Bayes twoaction problems I: Discrete case, Ann. Math. Statist. 42 (1971), 1521-1539.

[3] -, 一, Convergence rates for empirical Bayes two-action problems II: Continuous case, ibid. 43 (1972), 934-937.

[4] R. J. Karunamuni and H. Yang, On convergence rates of monotone empirical Bayes tests for the continuous one-parameter exponential family, Statist. Decisions 13 (1995), 181-192.

[5] T. Liang, On empirical Bayes tests in a positive exponential family, J. Statist. Plann. Inference 83 (2000), 169-181.

[6] H. Robbins, An empirical Bayes approach to statistics, in: Proc. Third Berkeley Sympos. Math. Statist. Probab., Univ. of California Press, 1955, 157-163.

[7] -, The empirical Bayes approach to statistical decision problems, Ann. Math. Statist. 35 (1964), 1-20.

[8] Th. Stijnen, On the asymptotic behaviour of empirical Bayes tests for the continuous one-parameter exponential family, Ann. Statist. 13 (1985), 403-412.

[9] J. C. van Houwelingen, Monotone empirical Bayes tests for the continuous oneparameter exponential family, ibid. 4 (1976), 981-989.

[10] S. P. Zhang and R. J. Karunamuni, Empirical Bayes estimation for the continuous one-parameter exponential family with error in variables, Statist. Decisions 15 (1997), 261-279.

[11] - - - Bayes and empirical Bayes estimation with errors in variables, Statist. Probab. Lett. 33 (1997), 23-34.

Limburgs Universitair Centrum

Center for Statistics

B-3590 Diepenbeek, Belgium

E-mail: lichun.wang@luc.ac.be

Received on 29.7.2003;

revised version on 14.12.2004 